\title{
Algorithm for finding location domination number of Corona product of graphs
}

\author{
G. Rajasekar ${ }^{1 *}$ and K. Nagarajan ${ }^{2}$
}

\begin{abstract}
A dominating set $S$ which uniquely identify all vertices of the set $V(G)-S$ is a locating dominating set. This paper presents the procedure for finding the location domination number of Corona product of graphs. And location domination number of graph of forms $G \odot K_{n}, G \odot K_{m, n}, G \odot \bar{K}_{n}, G \odot S_{n}, G \odot P_{n}, G \odot C_{n}, G \odot W_{n}$ and $G \odot F_{1, n}$ are deduced.

Keywords

Dominating set, Locating set, Locating domination set, Corona product.

\section{AMS Subject Classification} 05C69, $05 \mathrm{C} 76$.

1,2 Department of Mathematics, Jawahar Science College, Neyveli-607803, India.

*Corresponding author: ${ }^{\text {}}$ grsmaths@gmail.com; ${ }^{2}$ n2a4g1r1j1@gmail.com

Article History: Received 24 March 2019; Accepted 09 May 2019

(C)2019 MJM.
\end{abstract}

\section{Contents}

1 Introduction 130

2 Location Domination Number of Corona Product of Graphs ................................. 130

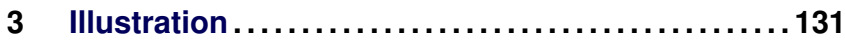

$3.1 G \odot K_{1} \ldots \ldots \ldots \ldots \ldots \ldots \ldots \ldots \ldots \ldots \ldots \ldots$

$3.2 G \odot K_{m, n} \ldots \ldots \ldots \ldots \ldots \ldots \ldots \ldots \ldots \ldots \ldots \ldots \ldots$

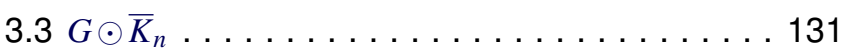

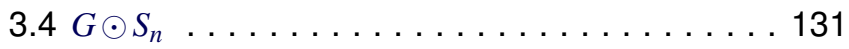

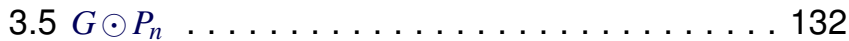

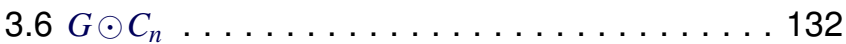

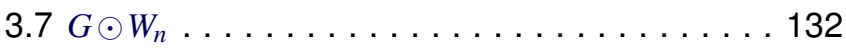

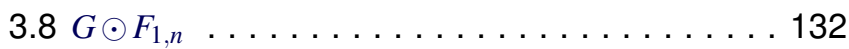

4 Conclusion. 133

References 133

\section{Introduction}

Oystein Ore [2] defined that the dominating set of a graph $G$ is a subset $\mathrm{S}$ of the vertex set $V(G)$ such that all vertices in the set $V(G)-S$ is adjacent to atleast one vertex in $S$. Minimal cardinality of dominating set is known as domination number and it is denoted by $\gamma(G)$.

Slater $[4,5]$ defined the locating dominating set is a dominating set $S$ with $S(v) \neq S(w)$, for any $v, w \in V(G)-S$, where
$S(v)$ is the set of vertices in $S$ which are adjacent to $v$. A locating dominating set is denoted by $L D$-set. The minimum cardinality of an $L D$-set in $G$ is called the location-domination number of $G$ and it is denoted by $R D(G)$. An $L D$-set with $R D(G)$ elements is called as a referencing-dominating set or an $R D$-set.

In [1, 3] Sergio R. Canoy, Jr. et al. and Risan Nur Santi et al. have found the bound for location domination number of corona product of graphs. In this paper, we construct a systematic technique for finding location domination number of corona product of any graph and rectify some results in [3].

\section{Location Domination Number of Corona Product of Graphs}

In this section, we introduce a new definition "wholly located dominated graph" by which we determine location domination number of corona product of any graph.

Definition 2.1. Let $G$ be any graph with $R D$-set $S$. If $G$ has a vertex $v \in V(G)-S$, such that $S(v)=S$ then the vertex $v$ is said to be wholly located dominated vertex with respect to the $R D$ - set $S$.

Definition 2.2. Let $\mathscr{S}$ be the collection of all RD-set of $G$. Graph $G$ is said to be wholly located dominated graph iffor every $R D$-set $S \in \mathscr{S}$ there exist a wholly located dominated vertex. 
Theorem 2.3. Let $G_{1}$ and $G_{2}$ be any two graphs with vertex set $V\left(G_{1}\right)$ and $V\left(G_{2}\right)$ respectively. For the graph $G_{1} \odot G_{2}$, if $G_{2}$ wholly located dominated graph then

$$
R D\left(G_{1} \odot G_{2}\right)=R D\left(G_{2}\right)\left|V\left(G_{1}\right)\right|+\gamma\left(G_{1}\right)
$$

otherwise,

$$
R D\left(G_{1} \odot G_{2}\right)=R D\left(G_{2}\right)\left|V\left(G_{1}\right)\right|
$$

Proof.

Let $V\left(G_{1}\right)=\left\{u_{1}, u_{2}, \ldots, u_{m}\right\}$ and $V\left(G_{2}\right)=\left\{v_{1}, v_{2}, \ldots, v_{n}\right\}$, then $V\left(G_{1} \odot G_{2}\right)$ is

$$
\begin{aligned}
& \left\{u_{1}, u_{2}, \ldots, u_{m}\right\} \cup\left\{\left(u_{1}, v_{1}\right),\left(u_{1}, v_{2}\right), \ldots,\left(u_{1}, v_{n}\right)\right\} \cup \\
& \left\{\left(u_{2}, v_{1}\right),\left(u_{2}, v_{2}\right), \ldots,\left(u_{2}, v_{n}\right)\right\} \cup \ldots \cup \\
& \left\{\left(u_{m}, v_{1}\right),\left(u_{m}, v_{2}\right), \ldots,\left(u_{m}, v_{n}\right)\right\}
\end{aligned}
$$

By definition of corona product there are $\left|V\left(G_{1}\right)\right|$ copies of $G_{2}$ graph joined to each vertices of $G_{1}$. To locate dominate each copy of $G_{2}$, it requires $R D\left(G_{2}\right)$ number of vertices. Since there are $\left|V\left(G_{1}\right)\right|$ copies of $G_{2}$, it requires $R D\left(G_{2}\right)\left|V\left(G_{1}\right)\right|$ number of vertices to locate and dominate. Therefore $R D\left(G_{1} \odot G_{2}\right) \geq R D\left(G_{2}\right)\left|V\left(G_{1}\right)\right|$.

Let $S_{1}, S_{2}, \ldots, S_{m}$ be the set to locate and dominate the $m$ copies of the graph $G_{2}$. That is $S_{i}$ is the $R D$ set of the induced subgraph of $G_{1} \odot G_{2}$ with vertex set $\left\{\left(u_{i}, v_{1}\right),\left(u_{i}, v_{2}\right), \ldots,\left(u_{i}, v_{n}\right)\right\}$ where $i=1,2, \ldots, m$. Clearly $S_{i} \cap S_{j}=\Phi$ if $i \neq j$. Now consider the set $S=S_{1} \cup S_{2} \cup \ldots \cup S_{m}$. Case 1: Suppose $G_{2}$ is not wholly located dominated graph, then $G_{2}$ has atleast one $R D$-set $S_{G_{2}}$ such that $S_{G_{2}}(v) \neq S_{G_{2}}$ for all $v \in V\left(G_{2}\right)-S_{G_{2}}$. Hence in the graph $G_{1} \odot G_{2}$, $S\left(\left(u_{i}, v_{j}\right)\right)=S_{i}\left(v_{j}\right) \neq S_{i}$ and $S\left(u_{i}\right)=S_{i}$.

Clearly $S_{i}$ locate and dominate all vertices of the set $\left\{\left(u_{i}, v_{1}\right),\left(u_{i}, v_{2}\right), \ldots,\left(u_{i}, v_{n}\right)\right\}-S_{i}$ uniquely and it also uniquely locate and dominate the vertex $u_{i}$. So all the vertices of $\left(G_{1} \odot G_{2}\right)-S$ are uniquely located and dominated by the set $S$ and it is minimal. Hence,

$$
\begin{aligned}
R D\left(G_{1} \odot G_{2}\right)= & |S|=\left|S_{1} \cup S_{2} \cup \ldots \cup S_{m}\right| \\
= & \left|S_{1}\right|+\left|S_{2}\right|+\ldots+\left|S_{m}\right|, \\
& \quad \text { as } S_{i} \cap S_{j}=\Phi \text { if } i \neq j \\
= & m R D\left(G_{2}\right) \\
= & R D\left(G_{2}\right)\left|V\left(G_{1}\right)\right| .
\end{aligned}
$$

Case 2: Suppose assume that $G_{2}$ is wholly located dominated graph, then for $R D$-set $S_{G_{2}}$ of $G_{2}$ there exist every some vertex $v_{j} \in V\left(G_{2}\right)-S_{G_{2}}$ such that $S_{G_{2}}\left(v_{j}\right)=S_{G_{2}}$.

Now for the graph $G_{1} \odot G_{2}$, if we look upon the set $S=S_{1} \cup S_{2} \cup \ldots \cup S_{m}$, it clear that $S_{i}$ will be the $R D$-set of $i^{\text {th }}$ copy of $G_{2}$ in the graph $G_{1} \odot G_{2}$. By our assumption there exist a vertex $\left(u_{i}, v_{j}\right)$, where $i=1,2, \ldots, m$ such that $S\left(\left(u_{i}, v_{j}\right)\right)=S_{i}\left(v_{j}\right)=S_{i}$ and $S\left(u_{i}\right)=S_{i}$. Hence $S$ could not be the $L D$-set of $G_{1} \odot G_{2}$. But $S$ dominates all the vertices of $G_{1} \odot G_{2}$ and locate all the vertices uniquely except $u_{1}, u_{2}, \ldots, u_{m}$
As $S\left(u_{i}\right)=S_{i}$, it is enough to dominate the vertices $u_{1}, u_{2}, \ldots, u_{m}$ with the help of the vertices in the set $\left\{u_{1}, u_{2}, \ldots, u_{m}\right\}$, so that $S\left(u_{i}\right) \supset S_{i}$ and it would be the $L D$ set of $G_{1} \odot G_{2}$ and could not be further reduced.

Therefore $R D$-set of $G_{1} \odot G_{2}$ is the set $S$ union dominating set of $\left\{u_{1}, u_{2}, \ldots, u_{m}\right\}$. That is, $S$ union dominating set of $G_{1}$ is the $R D$-set of $G_{1} \odot G_{2}$. Hence,

$$
\begin{aligned}
R D\left(G_{1} \odot G_{2}\right) & =|S|+\gamma\left(G_{1}\right) \\
& =R D\left(G_{2}\right)\left|V\left(G_{1}\right)\right|+\gamma\left(G_{1}\right) .
\end{aligned}
$$

Remark 2.4. Corona product of two graphs is not commutative. Therefore $R D\left(G_{1} \odot G_{2}\right)$ need not be equal to $R D\left(G_{2} \odot G_{1}\right)$.

Remark 2.5. All disconnected graphs will not be a wholly located dominated graph. In the graph $G_{1} \odot G_{2}$, if $G_{2}$ is disconnected then it will not a wholly located dominated graph. Thus $R D\left(G_{1} \odot G_{2}\right)=R D\left(G_{2}\right)\left|V\left(G_{1}\right)\right|$.

\section{Illustration}

\section{$3.1 G \odot K_{1}$}

Corollary 3.1. As $K_{n}$ is not wholly located dominated graph when $n=1, R D\left(G \odot K_{1}\right)=|V(G)|$. For $n>1, K_{n}$ are all wholly located dominated graph so

$$
\begin{aligned}
R D\left(G \odot K_{n}\right) & =R D\left(K_{n}\right)|V(G)|+\gamma(G) \\
& =(n-1)|V(G)|+\gamma(G) .
\end{aligned}
$$

$3.2 G \odot K_{m, n}$

Corollary 3.2. For $m, n \geq 2, K_{m, n}$ are all not wholly located dominated graph. Hence

$$
\begin{aligned}
R D\left(G \odot K_{m, n}\right) & =R D\left(K_{m, n}\right)|V(G)| \\
& =(m+n-2)|V(G)| .
\end{aligned}
$$

$3.3 G \odot \bar{K}_{n}$

Corollary 3.3. $\bar{K}_{n}$ is not wholly located dominated graph for all $n \geq 1$. By Theorem 2.3 we obtain

$$
\begin{aligned}
R D\left(G \odot \bar{K}_{n}\right) & =R D\left(\bar{K}_{n}\right)|V(G)| \\
& =n|V(G)| .
\end{aligned}
$$

$3.4 G \odot S_{n}$

Result 3.1. Consider the star graph $S_{n}=K_{1, n}$ as shown in Fig. 1. It's vertex set be $\left\{u, v_{1}, v_{2}, \ldots, v_{n}\right\}$, where $\operatorname{deg}(u)=n$ and $\operatorname{deg}\left(v_{i}\right)=1$ for $i=1,2, \ldots, n$.

Clearly $S_{1}=K_{1,1}=K_{2}$ is a wholly located dominated graph. Any set with cardinality $n$ will be the $R D$-set of $S_{n}$. Therefore $S=\left\{u, v_{1}, v_{2}, \ldots, v_{n-1}\right\}$ will be the $R D$-set of $S_{n}$. For $n>1, S_{n}$ has the $R D$-set $S$ which has no wholly located dominated vertex. Hence $S_{n}, n>1$ are all not wholly located dominated graph. 


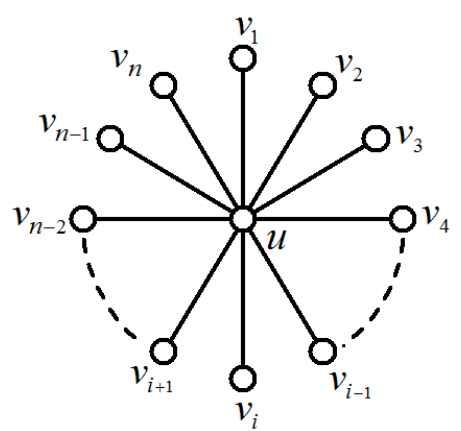

Figure 1. Star Graph $S_{n}$

Corollary 3.4. For the graph $S_{n}$, by Theorem 2.3 and Result 3.1 we get

$$
R D\left(G \odot S_{1}\right)=R D\left(S_{1}\right)|V(G)|+\gamma(G)=|V(G)|+\gamma(G)
$$

and for $n>1$,

$$
R D\left(G \odot S_{n}\right)=R D\left(S_{n}\right)|V(G)|=n|V(G)| .
$$

$3.5 G \odot P_{n}$

Result 3.2. Path graph $P_{2}, P_{5}$ are wholly located dominated graph while $P_{n}, n \neq 2,5$ are all not wholly located dominated graph.

Corollary 3.5. For the graph $P_{n}$, combining with the fact of Result 3.2 and Theorem 2.3 we get

$$
\begin{aligned}
R D\left(G \odot P_{n}\right) & = \begin{cases}R D\left(P_{n}\right)|V(G)|+\gamma(G), & \text { if } n=2,5 \\
R D\left(P_{n}\right)|V(G)|, & \text { otherwise }\end{cases} \\
& = \begin{cases}\left\lceil\frac{2 n}{5}\right\rceil|V(G)|+\gamma(G), & \text { if } n=2,5 \\
\left\lceil\frac{2 n}{5}\right\rceil|V(G)|, & \text { otherwise }\end{cases}
\end{aligned}
$$

$3.6 G \odot C_{n}$

Result 3.3. For the cycle graph $C_{n}, n \geq 3$ it is obvious that $C_{3}=K_{3}$ and $C_{5}$ are wholly located dominated graph and $C_{n}$, $n \neq 3,5$ they are all not wholly located dominated graph.

Corollary 3.6. For the cycle $C_{n}, n \geq 3$

$$
\begin{aligned}
R D\left(G \odot C_{n}\right) & = \begin{cases}R D\left(C_{n}\right)|V(G)|+\gamma(G), & \text { if } n=3,5 \\
R D\left(C_{n}\right)|V(G)|, & \text { otherwise }\end{cases} \\
& = \begin{cases}\left\lceil\frac{2 n}{5}\right\rceil|V(G)|+\gamma(G), & \text { if } n=3,5 \\
\left\lceil\frac{2 n}{5}\right\rceil|V(G)|, & \text { otherwise }\end{cases}
\end{aligned}
$$

3.7 $G \odot W_{n}$

Result 3.4. $W_{n}, n \geq 3$ is the wheel graph whose vertex set is $\left\{u, v_{1}, v_{2}, \ldots, v_{n}\right\}$ with $\operatorname{deg}(u)=n$ and $\operatorname{deg}\left(v_{i}\right)=3$ is shown in the Fig. 2.

Graph $W_{3}$ and $W_{4}$ are obviously wholly located dominated graph. While $W_{5}$ has a $R D$-set $\left\{u, v_{1}, v_{2}\right\}$ which does not has a wholly located dominated vertex. Hence $W_{5}$ is not a wholly located dominated graph.

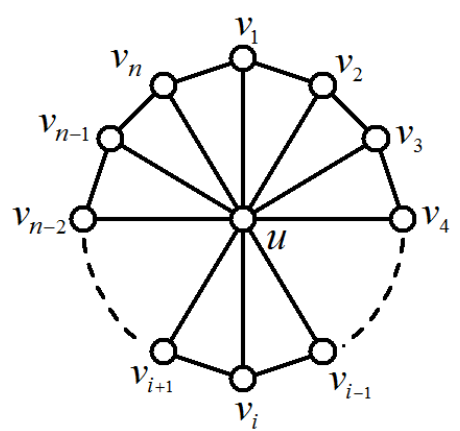

Figure 2. Wheel Graph $W_{n}$

If $W_{6}$ has a $R D$-set containing $\{u\}$ then it must also contain two vertices from the set $\left\{v_{1}, v_{2}, v_{3}, v_{4}, v_{5}\right\}$ in such a way that it locate and dominates 3 vertices, so there exist a wholly located dominated vertex. If $\{u\}$ does not belongs to the $R D$ set, then $u$ will be the wholly located dominated vertex. Hence in all possible $R D$ - set there exist a wholly located dominated vertex. So $W_{6}$ is a wholly located dominated graph.

For $n>6$, if $n \equiv 1 \bmod 5$ that is $n=5 k+1$ where $k \geq 2$, $W_{n}$ has a $R D$-set $\left\{u, v_{2}, v_{4}, v_{7}, v_{9}, \ldots, v_{5 k-3}, v_{5 k-1}\right\}$ without a wholly located dominated vertex. So $W_{n}$ is not a wholly located dominated graph when $n>6$ is $n \equiv 1 \bmod 5$.

Similarly when $n>6$ is $n \equiv 3 \bmod 5$, then $W_{n}$ is not a wholly located dominated graph with $R D$-set $\left\{u, v_{2}, v_{4}, v_{7}, v_{9}, \ldots, v_{5 k-3}, v_{5 k-1}, v_{5 k+1}\right\}$ which has no wholly located dominated vertex.

But when $n$ is of form $n=5(k+1)$ or $n=5 k+2$ or $n=5 k+4$ where $k \geq 1$, none of the $R D$-set contains $\{u\}$. But as $u$ is adjacent to all the vertices, it will definitely be the wholly located dominated vertex with respect to any $R D$-set. So for all $n>6, W_{n}$ will be the wholly located dominated graph when $n \equiv 0,2,4$ mod 5 .

Corollary 3.7. For wheel graph $W_{n}, n \geq 3$ by Theorem 2.3 and Result 3.4 we get

$$
R D\left(G \odot W_{n}\right)
$$

$= \begin{cases}R D\left(W_{n}\right)|V(G)|+\gamma(G), & \text { if } n=3,4,6 \text { or } \\ R D\left(W_{n}\right)|V(G)|, & \text { if } n>6, \text { and } n \equiv 0,2,4 \bmod 5 \\ \text { otherwise }\end{cases}$ $= \begin{cases}3|V(G)|+\gamma(G), & \text { if } n=3,6 \\ 2|V(G)|+\gamma(G), & \text { if } n=4 \\ 3|V(G)|, & \text { if } n=5 \\ \left\lceil\frac{2 n}{5}\right\rceil|V(G)|+\gamma(G), & \text { if } n>6 \text { and } n \equiv 0,2,4 \bmod 5 \\ \left\lceil\frac{2 n}{5}\right\rceil|V(G)|, & \text { if } n>6 \text { and } n \equiv 1,3 \bmod 5 .\end{cases}$

$3.8 G \odot F_{1, n}$

Result 3.5. Fan graph $F_{1, n}=K_{1}+P_{n}$ with vertex set $\left\{u, v_{1}, v_{2}, \ldots, v_{n}\right\}$ is shown in the Fig. 3 .

Clearly $F_{1,1}, F_{1,2}, F_{1,3}$ and $F_{1,4}$ are all wholly located dominated graph. $F_{1,5}$ has an $R D$-set $\left\{u, v_{3}, v_{4}\right\}$ with no wholly located dominated vertex. Hence $F_{1,5}$ is not a wholly located dominated graph. 


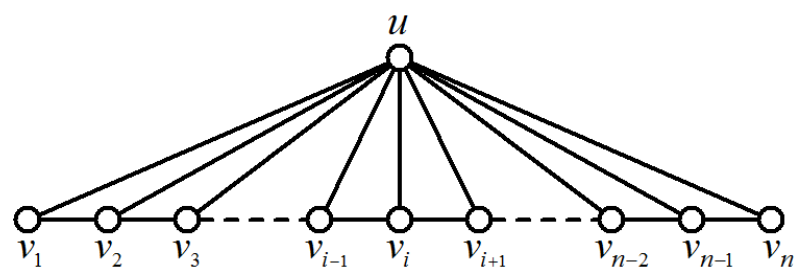

Figure 3. Fan Graph $F_{1, n}$

For the graph $F_{1,6}$ all possible $R D$-set has a wholly located dominated vertex. So $F_{1,6}$ should be a wholly located dominated graph.

Similar to $W_{n}$, we can show that $F_{1, n}$ is wholly located dominated graph when $n>6$ and $n \equiv 0,2,4 \bmod 5$.

For $n>6$, if $n \equiv 1 \bmod 5$, that is $n=5 k+1$ where integer $k \geq 2$, then we can find a $R D$-set $\left\{u, v_{2}, v_{4}, v_{7}, v_{9}, \ldots, v_{5 k-3}, v_{5 k-1}\right\}$ with no wholly located dominated vertex. Similarly for $n>6$, if $n \equiv 3 \bmod 5$ then $F_{1, n}$ has an $R D$-set $\left\{u, v_{2}, v_{4}, v_{7}, v_{9}, \ldots, v_{5 k-3}, v_{5 k-1}, v_{5 k+1}\right\}$ with no wholly located dominated vertex. Hence $F_{1, n}, n>6$ with $n \equiv 1,3 \bmod 5$ are all not wholly located dominated graph.

Corollary 3.8. Location domination number of the graph $G \odot F_{1, n}$ is given by

$$
R D\left(G \odot F_{1, n}\right)
$$

$= \begin{cases}R D\left(F_{1, n}\right)|V(G)|+\gamma(G), & \text { if } n=1,2,3,4,6 \text { or } \\ & \text { if } n>6 \text { and } n \equiv 0,2,4 \bmod 5 \\ R D\left(F_{1, n}\right)|V(G)|, & \text { otherwise }\end{cases}$ $= \begin{cases}|V(G)|+\gamma(G), & \text { if } n=1 \\ 2|V(G)|+\gamma(G), & \text { if } n=2,3,4 \\ 3|V(G)|, & \text { if } n=5 \\ 3|V(G)|+\gamma(G), & \text { if } n=6 \\ \left\lceil\frac{2 n}{5}\right\rceil|V(G)|+\gamma(G), & \text { if } n>6 \text { and } n \equiv 0,2,4 \bmod 5 \\ \left\lceil\frac{2 n}{5}\right\rceil|V(G)|, & \text { if } n>6 \text { and } n \equiv 1,3 \bmod 5 .\end{cases}$

Remark 3.9. By replacing graph $G$ in the Corollary 3.1, 3.2, 3.3, 3.4, 3.5, 3.6, 3.7, 3.8, with known graph we could directly find the location domination number of graph of form $G \odot K_{n}, G \odot K_{m, n}, G \odot \bar{K}_{n}, G \odot S_{n}, G \odot P_{n}, G \odot C_{n}, G \odot W_{n}$ and $G \odot F_{1, n}$.

\section{Conclusion}

In this paper by introducing wholly located dominated graph we have found location domination number of corona product of graphs. And we have deduced the location domination number of some general graphs like $G \odot K_{n}, G \odot K_{m, n}$, $G \odot \bar{K}_{n}, G \odot S_{n}, G \odot P_{n}, G \odot C_{n}, G \odot W_{n}$ and $G \odot F_{1, n}$.

\section{Acknowledgment}

This research is supported by UGC scheme RGNF. Award letter F1-17.1/2014-15/RGNF-2014-15-SC-TAM-80373/(SAIII/ Website).

\section{References}

[1] S. R. Canoy, Jr. and G. A. Malacas, Locating-dominating sets in graphs, App. Math. Sci., 8 (88) (2014), 4381-4388.

[2] O. Ore, Theory of Graphs, Amer. Math. Soc. Colloq. Publ., 38 (Amer. Math. Soc., Providence, RI), 1962.

[3] R. N. Santi, I. H. Agustin, Dafik and R. Alfarisi, On the locating domination number of corona product, J. Phys. Conf. Ser., 1008 (2018), 012053.

[4] P. J. Slater, Dominating and reference sets in a graph, $J$. Math. Phys. Sci., 22 (1988), 445-455.

[5] P. J. Slater, Dominating and location in acyclic in graphs, Networks, 17 (1987), 55-64.

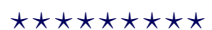

$$
\begin{aligned}
& \text { ISSN(P):2319 - } 3786 \\
& \text { Malaya Journal of Matematik } \\
& \text { ISSN(O):2321 - } 5666
\end{aligned}
$$

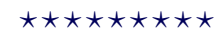

